\title{
Lattice stretching bistability and dynamic heterogeneity
}

\section{Christiansen, Peter Leth; Savin, A. V.; Zolotaryuk, A. V.}

\section{Published in:}

Physical Review E

Link to article, DOI:

10.1103/PhysRevE.85.046601

Publication date:

2012

\section{Document Version}

Publisher's PDF, also known as Version of record

Link back to DTU Orbit

Citation (APA):

Christiansen, P. L., Savin, A. V., \& Zolotaryuk, A. V. (2012). Lattice stretching bistability and dynamic heterogeneity. Physical Review E, 85(4), 046601. https://doi.org/10.1103/PhysRevE.85.046601

\section{General rights}

Copyright and moral rights for the publications made accessible in the public portal are retained by the authors and/or other copyright owners and it is a condition of accessing publications that users recognise and abide by the legal requirements associated with these rights.

- Users may download and print one copy of any publication from the public portal for the purpose of private study or research.

- You may not further distribute the material or use it for any profit-making activity or commercial gain

- You may freely distribute the URL identifying the publication in the public portal

If you believe that this document breaches copyright please contact us providing details, and we will remove access to the work immediately and investigate your claim 


\title{
Lattice stretching bistability and dynamic heterogeneity
}

\author{
P. L. Christiansen \\ Department of Informatics and Department of Physics, Technical University of Denmark, DK 2800, Kgs. Lyngby, Denmark \\ A. V. Savin \\ Semenov Institute of Chemical Physics, Russian Academy of Sciences, Moscow 119991, Russia
}

A. V. Zolotaryuk

Bogolyubov Institute for Theoretical Physics, National Academy of Sciences of Ukraine, Kyiv 03680, Ukraine

(Received 3 November 2011; revised manuscript received 20 February 2012; published 2 April 2012)

\begin{abstract}
A simple one-dimensional lattice model is suggested to describe the experimentally observed plateau in force-stretching diagrams for some macromolecules. This chain model involves the nearest-neighbor interaction of a Morse-like potential (required to have a saturation branch) and a harmonic second-neighbor coupling. Under an external stretching applied to the chain ends, the intersite Morse-like potential results in the appearance of a double-well potential within each chain monomer, whereas the interaction between the second neighbors provides a homogeneous bistable (degenerate) ground state, at least within a certain part of the chain. As a result, different conformational changes occur in the chain under the external forcing. The transition regions between these conformations are described as topological solitons. With a strong second-neighbor interaction, the solitons describe the transition between the bistable ground states. However, the key point of the model is the appearance of a heterogenous structure, when the second-neighbor coupling is sufficiently weak. In this case, a part of the chain has short bonds with a single-well potential, whereas the complementary part admits strongly stretched bonds with a double-well potential. This case allows us to explain the existence of a plateau in the force-extension diagram for DNA and $\alpha$-helix protein. Finally, the soliton dynamics are studied in detail.
\end{abstract}

DOI: 10.1103/PhysRevE.85.046601

PACS number(s): 05.45.Yv, 63.20.Ry, 45.90.+t

\section{INTRODUCTION}

In the past decade new methods appeared which made it possible to stretch a single molecule and measure a stretching force with high precision. In these experiments one end of the molecule is attached to the surface, whereas another one is attached to a latex bead which is pulled by optical or magnetic tweezers [1-4]. The main result of these experiments is a force-extension diagram. Complex behavior has been revealed by elasticity studies of individual double-stranded DNA molecules. The force-extension diagram appeared here to have an unexpected structure - there exists a large interval of extensions at which the lengthening of a molecular chain is not accompanied by growth of its tension [5]. In this case, a plateau appears on the force-extension diagram. This behavior can be explained by only some structural changes of the chain under its stretching. In this regard, it was suggested that in the plateau region, the transition of DNA double helix from the canonical $B$ form into some new structure takes place. Therefore various models for the structure of the so-called $S$ form of DNA have been proposed, but so far its nature remains obscured. Single molecule stretching experiments have been carried out also for polypeptide molecules [6-8]. Similar diagrams with a typical force-extension plateau have been observed experimentally for synthetical $\alpha$ helices [7] and myosin molecules [6]. All these studies raise the question on the conformational changes of molecular chains under their stretching.

It is shown in this paper that within a simple onedimensional chain model with nearest-neighbor interaction in the form of a Morse-like potential and the second-neighbor harmonic interaction, only a soft anharmonicity of the intermolecular interaction can lead to essential conformational changes of the chain under its stretching, when one part of chain bonds appears to be weakly stretched, whereas the other one is strongly stretched. Of course, in the framework of this model it is impossible to explain rigorously the structural changes of the DNA double helix and $\alpha$ helix of protein under their stretching (for this purpose more sophisticated models that account for molecular structure have to be elaborated). Our aim here is to describe only on a qualitative level all possible conformational changes of the chain under its stretching. These changes are caused by soft anharmonicity of the nearestneighbor intermolecular interaction (more precisely, by the presence of a point of inflection in the interaction potential). There can be no doubt that the interactions of this type are present in strands of DNA and $\alpha$ helices of protein, and just the nonuniform stretching of bonds explains the structural changes of helical molecules resulting in the appearance of a plateau in the force-extension diagram.

The appearance of bistability under the chain stretching, when the whole chain or its part can be found in different ground states, has to cause the formation of topological defects (solitons) that describe the transition regions between these different conformations (ground states) in the chain. We will show that these defects are topological solitons that can propagate along the chain with subsonic velocities. At the end of this paper, some examples of extended macromolecules will be considered, where the anomalous behavior related to the appearance of bistability can be expected.

The effect of appearance of bistable states caused by lattice stretching has been first studied by Manevitch et al. [9] for modeling the mechanodestruction of a polymer chain. As a simple model, the anharmonic chain with the nearestneighbor coupling in the form of the Morse potential has been 

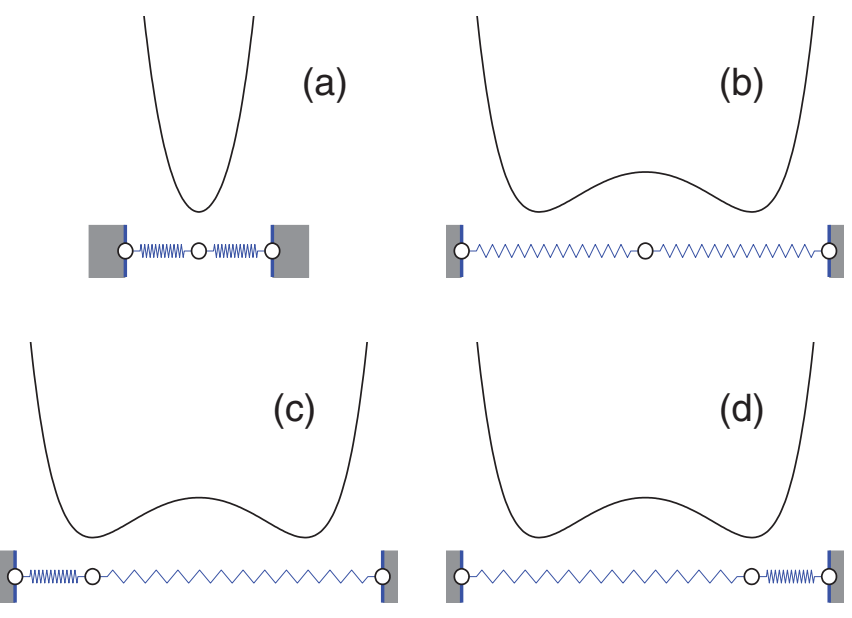

FIG. 1. (Color online) (a) Single-well and (b)-(d) double-well potentials for a central particle interacting with its fixed neighbors via interatomic Morse-like potential $V(r)$ and formed as the sum $V(r)+V(2 R-r)$. (a) Distance $2 R$ between the lateral fixed particles does not exceed $2 R_{0}$, where $R_{0}$ is a point of inflection of $V(r)$. (b) Unstable equilibrium position of the central particle. (c) Left and (d) right stable equilibria of the central particle.

chosen. Under lengthening this chain by applying an external force to its ends, the formation of an effective double-well potential in the chain bonds has been shown. The idea of this conformational transition can be explained in simple terms as follows. Consider three coupled particles as shown in Fig. 1, where the two lateral particles are fixed and the central particle interacts with its neighbors through a Morse-like potential $V(r)$ with a minimum at $R=r_{0}$ and a constant asymptote $\varepsilon=\lim _{r \rightarrow \infty} V(r)$. The potential of this type has a point of inflection at $r=R_{0}>r_{0}$. The Morse potential

$$
V(r)=\varepsilon\left[e^{-\beta\left(r-r_{0}\right)}-1\right]^{2}, \quad 0<r<\infty,
$$

given in dimensionless units, with any parameter $\beta>0$, can be chosen as a particular example where $R_{0}=r_{0}+\beta^{-1} \ln 2$. The total potential for the middle particle is $V(r)+V(2 R-r)$, where $2 R$ is the distance between the lateral particles. Using the new variable $u=R-r$, this potential can be written in a more convenient symmetric form as follows:

$$
\begin{aligned}
W(u) \doteq & V(R-u)+V(R+u)=\varepsilon e^{-2 \beta\left(R-r_{0}\right)} \\
& \times\left\{\left[2 \cosh (\beta u)-e^{\beta\left(R-r_{0}\right)}\right]^{2}+e^{2 \beta\left(R-r_{0}\right)}-2\right\} .
\end{aligned}
$$

The potential $W(u)$ has only one minimum $u=0$ if $R \leqslant R_{0}$ as demonstrated by Fig. 1(a), and one maximum $u=0$ and the two minima $u= \pm u_{0}$ if $R>R_{0}$ [see Figs. 1(b)-1(d)]. For the potential $(1), u_{0}(R)=\beta^{-1} \operatorname{arccosh}\left[e^{\beta\left(R-r_{0}\right)} / 2\right]>0$, being the solution of the equation

$$
\cosh \left(\beta u_{0}\right)=e^{\beta\left(R-r_{0}\right)} / 2 .
$$

The three-particle system illustrated by Fig. 1 can be extended to a finite chain consisting of $2 N+1$ particles (or $N$ monomers), where the terminal particles are fixed and the total chain length is $L=2 N R$. If $R>R_{0}$, double-well potentials can be formed inside the chain. In this case, many ground states of the chain are possible resulting in different (a)

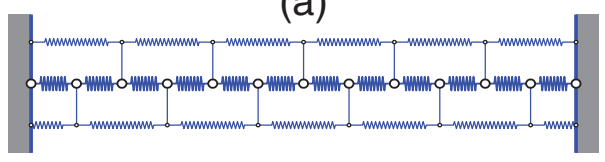

(b)

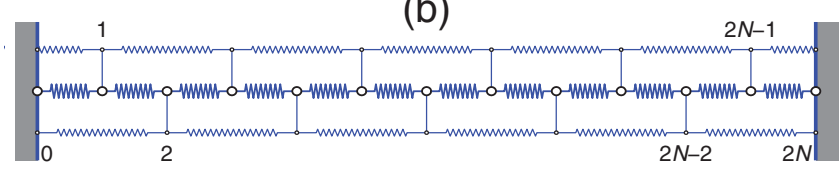

(c)

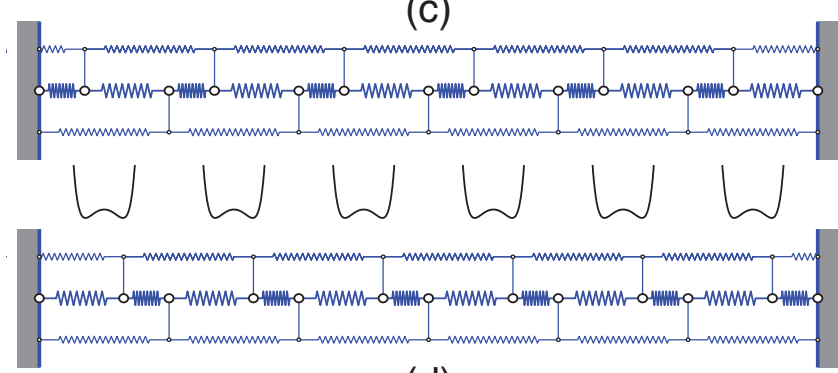

(d)

FIG. 2. (Color online) Equilibria in monoatomic chain with fixed terminal particles, where except for the nearest-neighbor interactions, also a coupling between the second neighbors is involved: (a) Stable (ground) state of the stretched chain with period $R \leqslant R_{0}$, where each atom is found in a single-well potential. (b) Unstable state of the chain, where all atoms with odd number are located at the top of a double-well potential. (c) Left and (d) right degenerate ground states of the stretched chain.

irregular chain conformations. Therefore the model studied in Ref. [9] has to be modified in such a way that a sufficiently stretched chain would admit homogeneous ground states with periodic structure. To this end, we involve additionally a stabilizing second-neighbor interaction and, as a result, the ground states with alternating lengths of chain bonds are possible.

It is sufficient to impose a harmonic coupling between the second neighbors as shown schematically in Fig. 2. Let $K$ be a (dimensionless) stiffness constant of the second-neighbor interaction with $x_{0}, x_{1}, \ldots, x_{2 N}$ being positions of the chain atoms. Then the total potential energy of the $N$-monomer system with fixed terminal atoms can be written as

$$
\begin{aligned}
E_{N}= & \frac{1}{2} \sum_{n=0}^{2 N-1} V\left(x_{n+1}-x_{n}\right)+K\left(x_{1}-x_{0}-r_{0}\right)^{2} \\
& +\sum_{n=0}^{2 N-2} \frac{1}{2} K\left(x_{n+2}-x_{n}-2 r_{0}\right)^{2} \\
& +\frac{1}{2} K\left(x_{2 N}-x_{2 N-1}-r_{0}\right)^{2} .
\end{aligned}
$$

It is expected that a sufficiently strong stretching of the chain results in a dimerization of the chain, for which the even atoms are found in equilibria $x_{n}=n R$ with $n=0,2, \ldots, 2 N$. 
Inserting these values into the energy (4), we find

$$
\begin{aligned}
E_{N}= & N[V(r)+V(2 R-r)]+4(N-1) K\left(R-r_{0}\right)^{2} \\
& +\frac{1}{2} K\left(r-r_{0}\right)^{2}+\frac{1}{2} K\left(2 R-r-r_{0}\right)^{2} .
\end{aligned}
$$

Differentiating Eq. (5) with respect to $r$, we find the following equation for equilibria:

$$
V^{\prime}(r)-V^{\prime}(2 R-r)=(2 K / N)(R-r) .
$$

Using the variable $u=R-r$, Eq. (6) becomes $W^{\prime}(u)=$ $-2 K u / N$. The trivial solution $u=0$ describes equilibria of odd atoms (stable if $R \leqslant R_{0}$ and unstable if $R>R_{0}$ ). The two stable equilibria with $u= \pm u_{0}$ appear when $R>R_{0}$ and $N \gg 1$. For a long chain $(N \rightarrow \infty)$, the role of boundary conditions can be neglected, so that Eq. (6) for the equilibrium positions $\pm u_{0}$ in the case of the Morse potential (1) takes the form of Eq. (3), so that the two stable minima exist if the inequality $R>R_{0}$ is fulfilled.

To simplify the problem with fixed chain ends, it is convenient to use the cyclic boundary conditions by putting

$$
x_{2 N}=x_{0}+2 N R \text { and } x_{2 N+1}=x_{1}+2 N R .
$$

Then, if we fix the whole chain by setting $x_{0} \equiv 0$, so that $x_{1}= \pm u_{0}$, the equilibrium positions are

$$
x_{n}^{0}=\left\{\begin{array}{l}
n R \quad \text { if } \quad n=0,2, \ldots, 2 N, \\
n R \pm u_{0} \quad \text { if } \quad n=1,3, \ldots, 2 N+1,
\end{array}\right.
$$

The subscripts "+" and "-" denote the two degenerate ground states in the dimerized chain, respectively. Schematically, these states can be represented as $X-X-X-X-X \cdots X-X-$ $-X-X-X$ and $X-X-X-X-X \cdots X-X-X-$ $-X-X$, where the terminal $X$ 's are fixed and all the bulk atoms are found either in the left well or the right well, respectively. Obviously, the domain walls (topological kinks and antikinks) that separate these two ground states can be excited. However, this is true if the second-neighbor interaction is sufficiently strong, at least for the model suggested in this paper. As shown below, the situation appears more complicated for a weak second-neighbor coupling, the case being of experimental relevance for some macromolecules. More precisely, the existence of a plateau in the force-stretching diagrams for the DNA double helix [1-5] as well as for $\alpha$ helices of protein [10] can qualitatively be explained within the framework of our model.

The paper is organized as follows. In the next section, we present the equations of motion for a stretched nonlinear monoatomic chain and discuss the spectrum of smallamplitude oscillations. The analysis of switching a bistable ground state of the chain is given in Sec. III. In Sec. IV, we find the profiles of kink and antikink solutions and study their dynamical properties. The next section is devoted to realistic systems, where the topological soliton solutions obtained in the previous section are studied in detail. Conclusions are given in Sec. VI.

\section{A MODEL AND ITS LINEARIZED VERSION}

With the notations introduced in the previous section, the (dimensionless) Hamiltonian for the monoatomic chain model with the cyclic boundary conditions (7) can be written in the form

$$
\begin{aligned}
H= & \sum_{n=0}^{2 N-1}\left[\frac{1}{2} m \dot{x}_{n}^{2}+V\left(x_{n+1}-x_{n}\right)\right. \\
& \left.+\frac{1}{2} K\left(x_{n+2}-x_{n}-2 r_{0}\right)^{2}\right],
\end{aligned}
$$

where $m$ is a chain particle mass and the dot denotes the differentiation over time $t$. Here the strings connecting the second neighbors are assumed to be undistorted at length $2 r_{0}$. The corresponding equations of motion are

$$
\begin{aligned}
m \ddot{x}_{n}= & {\left[V^{\prime}\left(x_{n+1}-x_{n}\right)-V^{\prime}\left(x_{n}-x_{n-1}\right)\right] } \\
& +K\left(x_{n+2}-2 x_{n}+x_{n-2}\right),
\end{aligned}
$$

where $n=0,1, \ldots, 2 N-1$.

The linearized version of Eqs. (10) is obtained by putting $x_{n}(t)=x_{n}^{0}+v_{n}(t), n=0,1, \ldots, 2 N+1$, where the equilibria $x_{n}^{0}$ 's are defined by Eqs. (8). As a result, we find

$$
\begin{aligned}
m \ddot{v}_{n}= & {\left[k_{ \pm}\left(v_{n+1}-v_{n}\right)-k_{\mp}\left(v_{n}-v_{n-1}\right)\right] } \\
& +K\left(v_{n+2}-2 v_{n}+v_{n-2}\right),
\end{aligned}
$$

where $k_{ \pm}=V^{\prime \prime}\left(R \pm u_{0}\right)$. The upper subscript at the stiffness coefficient belongs to the particles with even (odd) $n$ 's and the lower one to those with odd (even) $n$ 's for the case when all the odd particles are found in the right (left) well of the double-well potential.

Under stretching $R \leqslant R_{0}$, the displacement $u_{0}$ becomes zero and therefore we have the stiffness constant $k=k_{-}=$ $k_{+}=V^{\prime \prime}(R)$. With the stretching of the chain, the stiffness $k(R)$ decreases monotonically and at $R=R_{0}$ it reaches zero (Fig. 3, curve 1). At further lengthening, the chain becomes bistable and the distance $2 u_{0}$ increases monotonically with the growth of $R$. The stiffness of the short bonds $k_{-}=\varepsilon V^{\prime \prime}\left(R-u_{0}\right)>0$ increases monotonically (Fig. 3, curve 3 ) and the stiffness of the long bonds becomes negative, $k_{+}=V^{\prime \prime}\left(R+u_{0}\right)<0$ (curve 2), but their sum is always

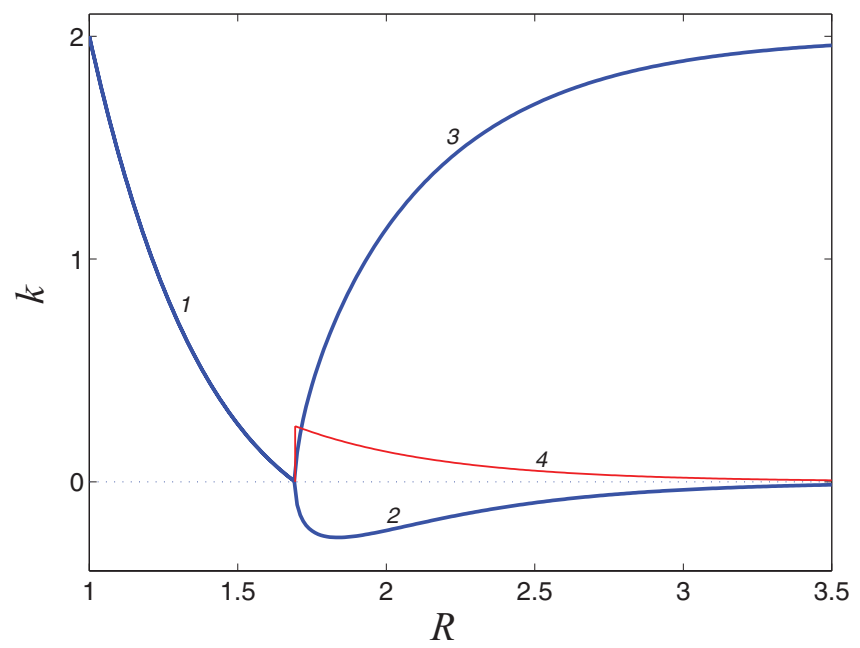

FIG. 3. (Color online) Stiffness $k=V^{\prime \prime}\left(R \pm u_{0}\right)$ as a function of lattice constant $R$ calculated for potential (4) with $\varepsilon=1, \beta=1$, and $r_{0}=1: k=k_{ \pm}$for $R \leqslant R_{0}$ (curve 1), $k=k_{+}$(curve 2), and $k=k_{-}$ (curve 3) for $R>R_{0}$. Critical stiffness $k=k_{c}=-k_{-} k_{+} / 2\left(k_{-}+k_{+}\right)$ is given by curve 4 . 
positive, $k_{-}+k_{+}>0$. Explicitly, for the potential (1) we get

$$
k_{ \pm}=\mp \varepsilon \beta^{2} e^{\mp \beta u_{0}} \sinh \left(\beta u_{0}\right) \cosh ^{-2}\left(\beta u_{0}\right),
$$

so that in this case $k_{-}+k_{+}=2 \varepsilon \beta^{2} \tanh ^{2}\left(\beta u_{0}\right)>0$.

The dispersion law is obtained if we insert the smallamplitude waves

$$
v_{n}(t)=A e^{i(q n R-\omega t)} \quad \text { and } \quad v_{n+1}(t)=B e^{i(q n R-\omega t)}
$$

into Eqs. (11) with $n=0,2, \ldots, 2 N$. Here $\omega$ is the frequency and the wave number $q \in[0, \pi / 2 R)$. As a result, we find the dispersion law that admits two branches of the spectrum (acoustic and optical):

$$
\begin{aligned}
m \omega_{ \pm}^{2}= & k_{-}+k_{+}+2 K[1-\cos (2 q R)] \\
& \pm \sqrt{k_{-}^{2}+k_{+}^{2}+2 k_{-} k_{+} \cos (2 q R)} .
\end{aligned}
$$

The dispersion curves for $R \leqslant R_{0}$ are present in Fig. 4(a). For $R<R_{0}$ the curve $\omega_{-}(q)$ is the continuation of the curve $\omega_{+}(q)$. They can be considered as a single acoustic branch. In the limit $R \rightarrow R_{0}-0$, the curves $\omega_{-}(q)$ and $\omega_{+}(q)$ merge together into a single curve $\omega^{2}=2 K[1-\cos (2 q R)]$. At further increase of $R$, this curve splits into two disconnected curves $\omega_{-}(q)$ and $\omega_{+}(q)$ [Fig. 4(b), curves 4 and 5], where the first curve corresponds to acoustic oscillations and the second one to optical oscillations of the stretched chain.

At stretching $R<R_{0}$, the uniformly stretched state is always stable, since all the coupling constants $k_{-}, k_{+}$, and $K$ are positive. The situation changes when $R>R_{0}$ because here one of the coupling constants, $k_{+}$, is negative and now the stability of the alternating state of the stretched chain depends on the stiffness constant $K$. For the stability it is necessary that

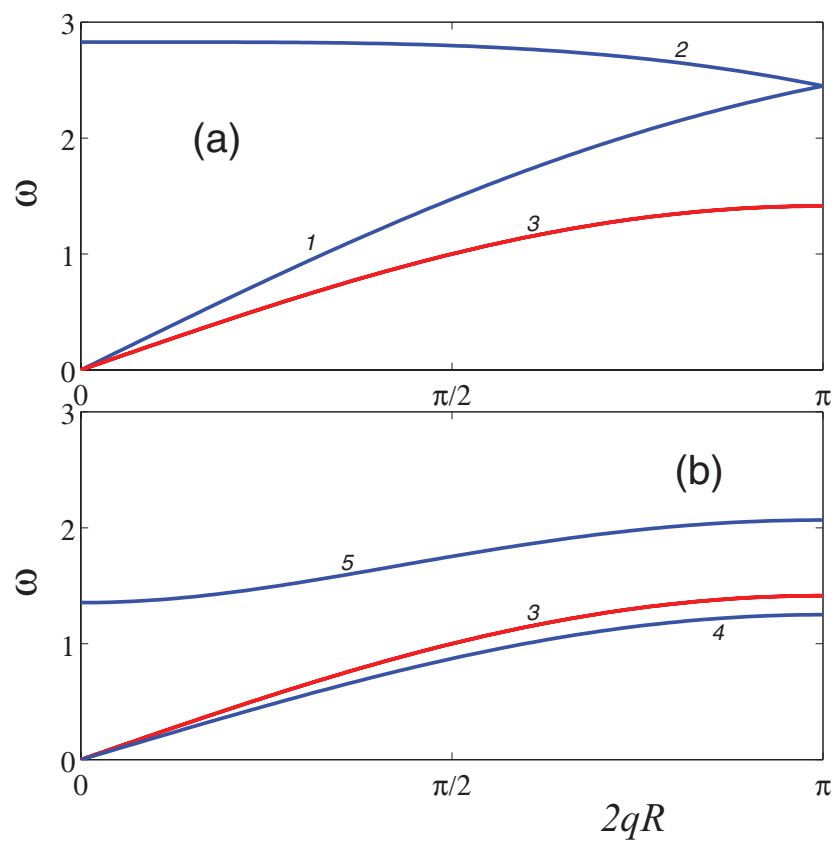

FIG. 4. (Color online) Linear spectra $\omega=\omega_{ \pm}(2 q R)$ calculated (at $m=1$ ) in the case of potential (1) with $\varepsilon=1, \beta=1, r_{0}=1$, and $K=0.5$ for (a) $R=r_{0}$ (curves 1 and 2), $R=R_{0}$ (curve 3), and (b) $R=2>R_{0}$ (curves 4 and 5). Curves 1,3 , and 4 relate to $\omega_{-}(2 q R)$ and curves 2,3 , and 5 relate to $\omega_{+}(2 q R)$. the inequality

$$
\begin{aligned}
& k_{-}+k_{+}+2 K[1-\cos (2 q R)] \\
& -\sqrt{k_{-}^{2}+k_{+}^{2}+2 k_{-} k_{+} \cos (2 q R)}>0
\end{aligned}
$$

has to be fulfilled for all values of the wave number $q>0$. It is easy to show that this condition holds only if

$$
K>k_{c}(R) \doteq-k_{-} k_{+} / 2\left(k_{-}+k_{+}\right) .
$$

The dependence of the critical value of the stiffness of the second-neighbor interaction on $R$ is given in Fig. 3 (curve 4). For all $R$ this value is positive; it monotonically decreases with increase of the chain stretching $R$. Its maximum reaches in the limit $R \rightarrow R_{0}+0$ when $u \rightarrow u_{0}+0$. Using Eq. (12), for the particular case (1) we obtain

$$
k_{c}(R)=\frac{\varepsilon \beta^{2}}{4} \cosh ^{-2}\left(\beta u_{0}\right)
$$

where the dependence of $u_{0}$ on $R$ is given by Eq. (3). Next, we find the limiting value $k_{0} \doteq \lim _{R \rightarrow R_{0}+0} k_{c}(R)=\varepsilon \beta^{2} / 4=$ $V^{\prime \prime}\left(r_{0}\right) / 8$ and therefore the stability condition of the alternating states of the stretched chain takes the following simple form:

$$
K>k_{0}=V^{\prime \prime}\left(r_{0}\right) / 8 .
$$

This relation of coupling constants for the interactions of the first and the second neighbors is a necessary and sufficient condition for the existence of stable stretched alternating states of the chain.

The velocity of long-wave acoustic phonons $v_{0}=$ $\lim _{q \rightarrow 0} \omega(q) / q$ can be calculated from the spectra (14) for different values of $R$. As a result, we obtain

$$
v_{0}=\left\{\begin{array}{l}
2 R \sqrt{(k / 4+K) / m} \quad \text { if } \quad R \leqslant R_{0}, \\
2 R \sqrt{\left(K-k_{c}\right) / m} \quad \text { if } \quad R>R_{0} .
\end{array}\right.
$$

Note that the condition (16) ensures the positivity of the expression under the radicals in (19).

\section{TRANSITION TO THE BISTABILITY OF THE GROUND STATE UNDER STRETCHING THE CHAIN}

In order to understand how the ground state of the chain changes under its stretching, we consider the dependence of the ground energy $E$ of the homogeneous chain state on the lattice spacing $R$. For the uniformly stretched chain state, when $x_{n+1}-x_{n}=R, 0<R<\infty, n=0,1, \ldots, 2 N$, the deformation energy of one chain unit is

$$
\begin{aligned}
E_{1}(R) & =V(R)+2 K\left(R-r_{0}\right)^{2} \\
& =\varepsilon\left[e^{-\beta\left(R-r_{0}\right)}-1\right]^{2}+2 K\left(R-r_{0}\right)^{2} .
\end{aligned}
$$

On the other hand, when we consider the ground state with the alternating bond lengths $R-u_{0}$ and $R+u_{0}\left(R>R_{0}\right)$, the deformation energy of one chain unit becomes

$$
\begin{aligned}
E_{2}(R) & =W\left(u_{0}\right) / 2+2 K\left(R-r_{0}\right)^{2} \\
& =\frac{\varepsilon}{2}\left[1-2 e^{-2 \beta\left(R-r_{0}\right)}\right]+2 K\left(R-r_{0}\right)^{2} .
\end{aligned}
$$

For comparison the form of the functions $E_{1}(R)$ and $E_{2}(R)$ is depicted in Fig. 5. The function $E_{1}(R)$ 

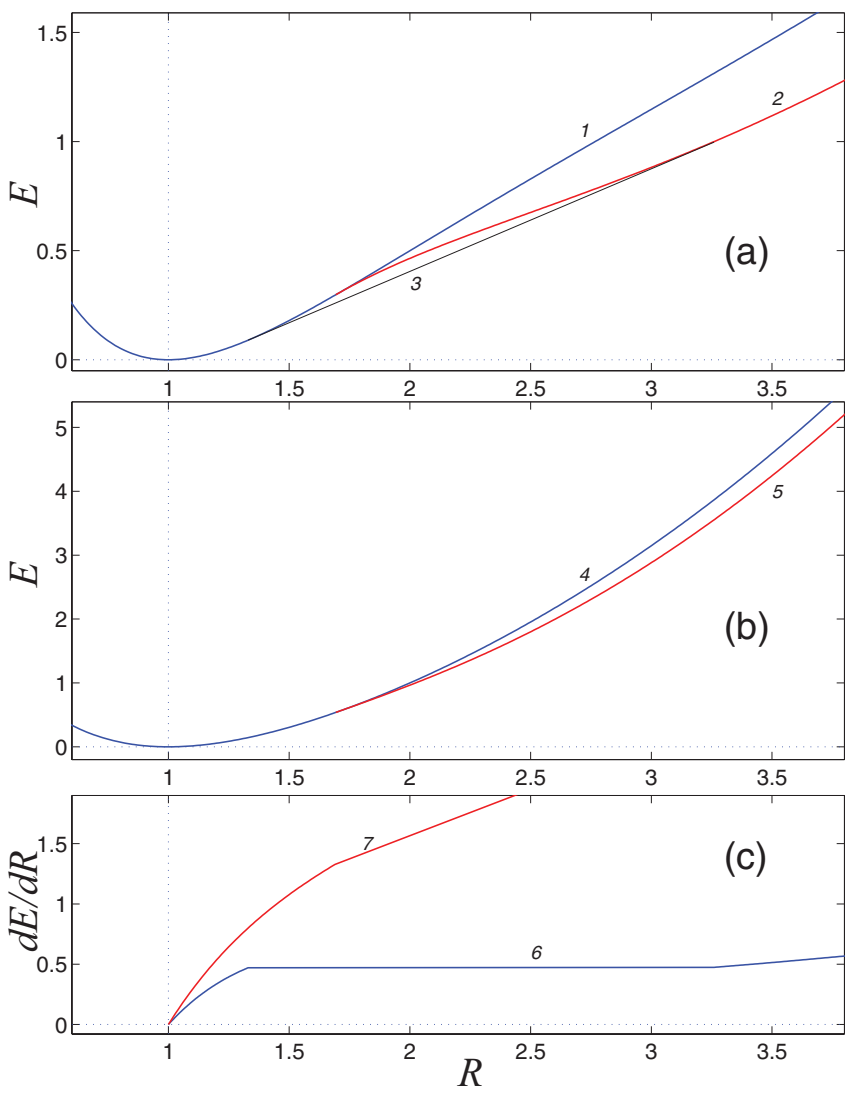

FIG. 5. (Color online) Dependence of energy $E$ and its derivative $d E / d R$ of uniformly stretched state of the chain with equal bonds $E=E_{1}$ (curves 1 and 4) and that with alternating bonds $E=E_{2}$ (curves 2 and 5) on lattice spacing $R$ for (a) and (c), line 6, $K=0.05$; and (b) and (c), line 7, $K=0.3\left(\beta=1, \varepsilon=1, r_{0}=1, K_{0}=0.25\right.$, and $R_{0}=1+\ln 2=1.693$ ). Lines 3 and 6 give convex neighborhood of function $E(R)$.

has a minimum at $R=r_{0}$, increasing for $R>r_{0}$. At $R=R_{0}$ both these functions are smoothly "sewed" together because $E_{1}\left(R_{0}\right)=E_{2}\left(R_{0}\right)=\varepsilon / 4+2 K\left(R_{0}-r_{0}\right)^{2}$ and $E_{1}^{\prime}\left(R_{0}\right)=E_{2}^{\prime}\left(R_{0}\right)=\varepsilon \beta / 2+4 K\left(R_{0}-r_{0}\right)$. However, for $R>$ $R_{0}$ the function $E_{2}(R)$ steps aside smoothly and continues further below $E_{1}(R)$. Therefore the energy of the homogeneously stretched (with any $R$ ) ground state of the chain is given by the smoothly sewed function $E(R)=E_{1}(R)$ for $R \leqslant R_{0}$ and $E(R)=E_{2}(R)$ for $R \geqslant R_{0}$. At $R=R_{0}$ the second derivative $E_{2}^{\prime \prime}\left(R_{0}\right)=4 K-\varepsilon \beta^{2}$ is positive if $K>K_{0}$ and negative if $K<K_{0}$. Therefore $E(R)$ is a strongly concave function (for all $R$ ) only if $K>K_{0}$ [see Fig. 13(b)]. In this case, the ground state of the chain is always the homogeneous conformation with equal bond lengths for $R \leqslant R_{0}$ and that with alternating bonds for $R>R_{0}$. The inequality (18) ensures the stability of the uniformly stretched state of the chain.

For $K<K_{0}$ a local convexity in the $E(R)$ behavior, as illustrated in Fig. 5(a) by curve 2, appears in a neighborhood of $R_{0}$, i.e., on some interval $R_{1}<R<R_{2}$ with $R_{1}<R_{0}$ and $R_{2}>R_{0}$. This means that the homogeneous state given by the energy $E_{2}(R), R_{1}<R<R_{2}$, with the alternating bond lengths $R-u_{0}(R)$ and $R+u_{0}(R)$, in fact is unstable. Instead, a heterogeneous conformation, where some part of the chain has equal bonds and the other one alternating bonds, appears more stable. In this case, the chain energy behavior can be obtained by connecting the two points $\left\{R_{1}, E\left(R_{1}\right)\right\}$ and $\left\{R_{2}, E\left(R_{2}\right)\right\}$ by a line [see Fig. 5(a), line 3]. In other words, moving along this line, the heterogeneous state with one part of the chain being in a weakly stretched state with equal bonds and the spacing $R_{1}$, and the other part in a strongly stretched state with alternating bond lengths $R_{2}-u_{0}\left(R_{2}\right), R_{2}+u_{0}\left(R_{2}\right)$ and the spacing $R_{2}$ appears more energetically favorable. In this case, the heterogeneous stretching (lengthening) of the whole chain occurs due to the increase of the portion of strongly stretched bonds. This scenario of the heterogeneous stretching results in the appearance of the stationary region (plateau) in the force-stretching diagram under the chain lengthening $\left(R_{1}<R<R_{2}\right)$ as illustrated by line 6 in Fig. 5(c).

\section{DYNAMIC HETEROGENEITY AND TOPOLOGICAL SOLITONS}

Since the nonlinear lattice model introduced in the previous section admits the heterogeneous structure that appears to be energetically favorable, the existence of freely moving topological defects is expected. The corresponding soliton solutions can be found numerically using the steepest-descent method. To this end, it is convenient to use the following variables: coordinates $u_{n}=x_{n} / r_{0}$, energy $\mathcal{H}=H / \varepsilon$, and time $\tau=r_{0}^{-1} \sqrt{\varepsilon / m} t$. Then the dimensionless Hamiltonian of the cyclic chain takes the form

$$
\begin{array}{r}
\mathcal{H}=\sum_{n=0}^{2 N-1}\left[\frac{1}{2}\left(\frac{d u_{n}}{d \tau}\right)^{2}+\mathcal{V}\left(u_{n+1}-u_{n}\right)\right. \\
\left.+\frac{1}{2} \kappa\left(u_{n+2}-u_{n}-2\right)^{2}\right],
\end{array}
$$

where $\mathcal{V}\left(u_{n+1}-u_{n}\right)=\varepsilon^{-1} V\left(x_{n+1}-x_{n}\right)$ and $\kappa=K r_{0}^{2} / \varepsilon$. According to Eqs. (7), the chain tension is given through the boundary conditions:

$$
u_{2 N} \equiv u_{0}+2 N a \quad \text { and } \quad u_{2 N+1} \equiv u_{1}+2 N a,
$$

where the lattice spacing of the stretched chain is $a=R / r_{0} \geqslant$ 1.

The system of equations

$$
\begin{aligned}
\frac{d^{2} u_{n}}{d \tau^{2}}= & \mathcal{V}^{\prime}\left(u_{n+1}-u_{n}\right)-\mathcal{V}^{\prime}\left(u_{n}-u_{n-1}\right) \\
& +\kappa\left(u_{n+2}-2 u_{n}+u_{n-2}\right)
\end{aligned}
$$

where $n=0,1, \ldots, 2 N-1$, corresponds to the Hamiltonian function (22) with the boundary conditions (23) and $u_{-1} \equiv$ $u_{2 N-1}-2 N a$ and $u_{-2} \equiv u_{2 N-2}-2 N a$. For the relative displacements $r_{n}=u_{n+1}-u_{n}$, the equations of motion (24) become

$$
\begin{aligned}
\frac{d^{2} r_{n}}{d \tau^{2}}= & \mathcal{V}^{\prime}\left(r_{n+1}\right)-2 \mathcal{V}^{\prime}\left(r_{n}\right)+\mathcal{V}^{\prime}\left(r_{n-1}\right) \\
& +\kappa\left(r_{n+2}-2 r_{n}+r_{n-2}\right)
\end{aligned}
$$

For numerics we choose the following values of the parameters: $\varepsilon=1, \beta=1$, and $r_{0}=1$. Then for $\kappa>\kappa_{0}=1 / 4$, the ground state will always be uniformly stretched because 
the stability condition of the alternating states (18) is fulfilled. Consider the chain with the lattice spacing $a>a_{0}=R_{0} / r_{0}=$ $1+\left(\beta r_{0}\right)^{-1} \ln 2$ and $\kappa>\kappa_{0}$. Then the chain has the following two ground states with equal energy: $r_{2 n-1}=a \mp \delta$ and $r_{2 n}=a \pm \delta, n=1, \ldots, N / 2$, where $\delta=u_{0} / r_{0}$. We look for traveling wave solutions of the equations of motion (25) that describe the smooth transition of the chain from one ground state to the other one, i.e., we put

$$
r_{2 n-1}(\tau)=r_{1}(2 n a-s \tau), \quad r_{2 n}(\tau)=r_{2}(2 n a-s \tau),
$$

where $s$ is a dimensionless traveling wave velocity. The numerical method for finding the soliton solutions of the equations of motion (25) is described in detail in Refs. [11,12].

Let $\left\{r_{n}\right\}_{n=1}^{2 N}$ be a soliton solution of the system equations of motion (25). Then it is possible to find the soliton energy

$$
\begin{aligned}
E= & \frac{1}{2} \sum_{n=1}^{N}\left\{\mathcal{V}\left(r_{2 n-1}\right)+\mathcal{V}\left(r_{2 n}\right)+\frac{1}{2} \kappa\left(1+\bar{s}^{2}\right)\right. \\
& \left.\times\left[\left(r_{2 n-1}+r_{2 n}-2\right)^{2}+\left(r_{2 n}+r_{2 n+1}-2\right)^{2}\right]\right\},
\end{aligned}
$$

where $\bar{s}=s / 2 a \sqrt{\kappa}$ is the reduced velocity and

$$
D=1+2\left[\sum_{n=1}^{N / 2}\left(n+\frac{1}{2}-\bar{n}\right) p_{n}\right]^{1 / 2}
$$

is its diameter. Here, the soliton center $\bar{n}$ is given by

$$
\bar{n}=\sum_{n=1}^{N / 2}\left(n+\frac{1}{2}\right) p_{n}
$$

and the sequence

$$
p_{n}=\left(r_{2 n+1}-r_{2 n-1}\right) / S, S=\sum_{n=1}^{N / 2}\left(r_{2 n+1}-r_{2 n-1}\right),
$$

determines the distribution of deformation along the chain.

The shape of the topological soliton is presented in Fig. 6. Panel (a) shows that the lengths of odd bonds $r_{2 n-1}$ have the kink shape, whereas the lengths of even bonds $r_{2 n}$ have the antikink shape (and vice versa for the antikink). Next, as shown in panel (b), the local compression of the chain takes place in the region of soliton localization.

The energy of formation of the kink-antikink pair can be defined as the difference $\Delta E=E-E_{0}$, where $E$ is the energy of the stationary kink-antikink pair in the cyclic chain and $E_{0}$ is the energy of the ground state of the chain at a given lattice spacing $a>a_{0}$. As shown in Fig. 7(a), the formation energy monotonically increases with the growth of the lattice spacing. Nearby the critical value of the lattice spacing $a_{0}$, the energy of formation becomes infinitesimal. When $a \rightarrow a_{0}+0$, the energy $\Delta E \rightarrow 0$ and the soliton diameter $D \rightarrow \infty$. With increasing $a$, the soliton diameter monotonically decreases down to the value $D=3.4$ [see Fig. 7(b)].

Consider the dependence of the energy and the diameter of the soliton on its velocity. We choose the values $\beta=1$, $r_{0}=1, a=1.7>a_{0}=1+\ln 2=1.693$, and $\kappa>\kappa_{0}=1 / 4$. It follows from Eq. (19) that the reduced dimensionless
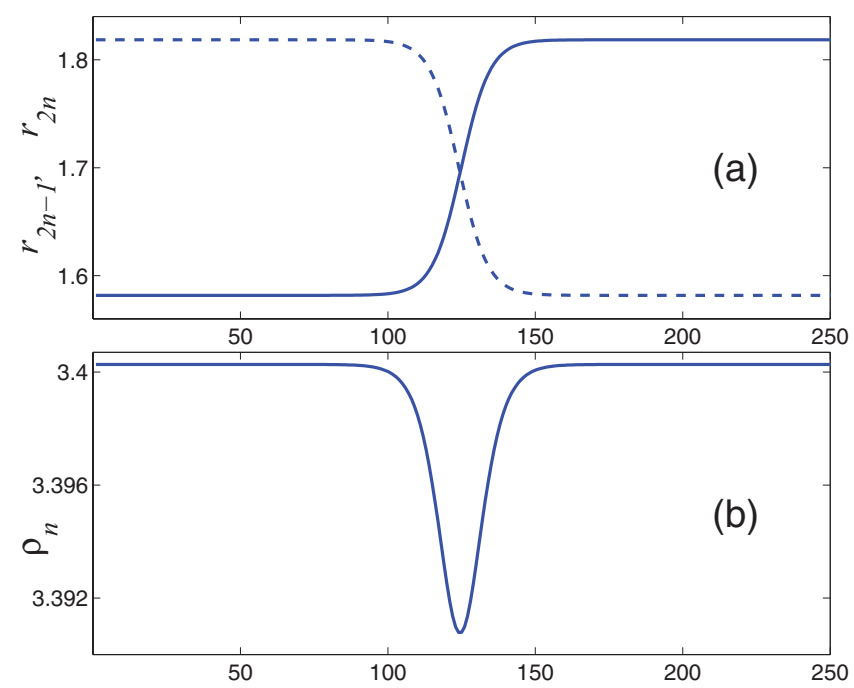

FIG. 6. (Color online) Stationary topological soliton $(s=0)$ in stretched chain at $a=1.7, \beta=1, r_{0}=1, \kappa=1, N=500$. Dependence of (a) relative displacements $r_{2 n-1}{ }^{*} r_{2 n}$ (solid and dashed lines) and (b) local compression $\rho_{n}=r_{2 n-1}+r_{2 n}$ on the number of the chain site are shown.

velocity of sound is

$$
\bar{s}_{0}=s_{0} / 2 a \sqrt{\kappa}=\sqrt{1+\frac{\kappa_{-} \kappa_{+}}{2 \kappa\left(\kappa_{-}+\kappa_{+}\right)}},
$$

where $\kappa_{ \pm}=V^{\prime \prime}(a \pm \delta)$. The numerical analysis has shown that the system of discrete equations (25) has a soliton solution only in the subsonic region: $\bar{s}<\bar{s}_{0}, \kappa>\kappa_{0}$, being a typical situation for topological solitons (kinks). For our model, the region of the existence of topological solitons in the space of the parameters $\kappa, \bar{s}$ is shown in Fig. 8. The region of the existence of solitons is separated from the region of their absence by
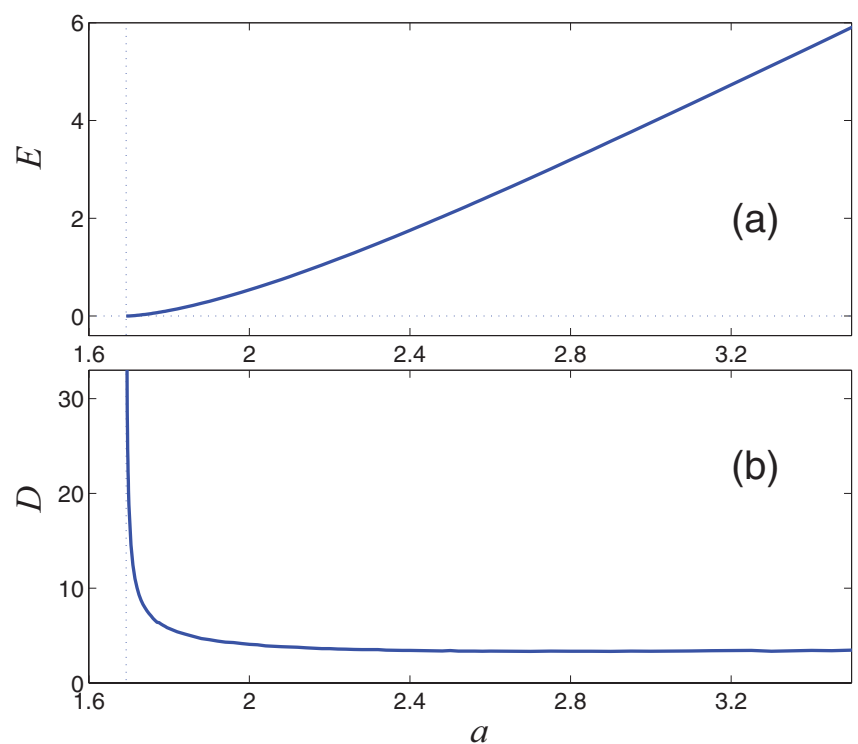

FIG. 7. (Color online) Dependence of the energy of formation of soliton-antisoliton pair $E$ (a) and the diameter of topological soliton $D$ (b) on the value of lattice spacing $a$ of stretched cyclic chain consisting of $N=500$ sites $\left(\beta=1, r_{0}=1, \kappa=1\right.$, and $\left.a_{0}=1.693\right)$. 


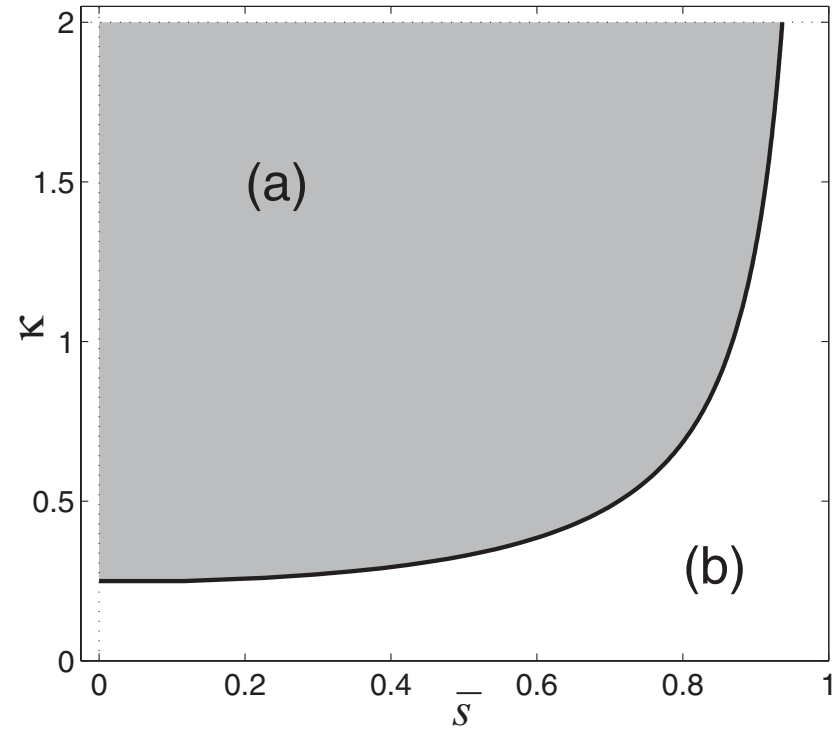

FIG. 8. Region of existence of topological solitons in the space of parameters $\bar{s}, \kappa$ (a) and region of parameter values at which soliton solutions are absent (b). The line which splits these regions is given by Eq. (31). Lattice spacing is $a=1.7$ and $\beta=1, r_{0}=1$.

curve (31), which determines the dependence of the reduced velocity of sound $\bar{s}_{0}$ on the dimensionless stiffness $\kappa$.

The dependence of the energy and the diameter of the topological soliton on its velocity is given in Fig. 9. As follows from this figure, the soliton energy monotonically increases with the growth the velocity. The energy tends to infinity at the velocity of long-wave acoustic phonons. The soliton diameter nonmonotonically depends on its velocity. For small values the velocity increase results in negligible decrease of the diameter, which nearby the right edge of the velocity spectrum turns into the fast monotonic growth.

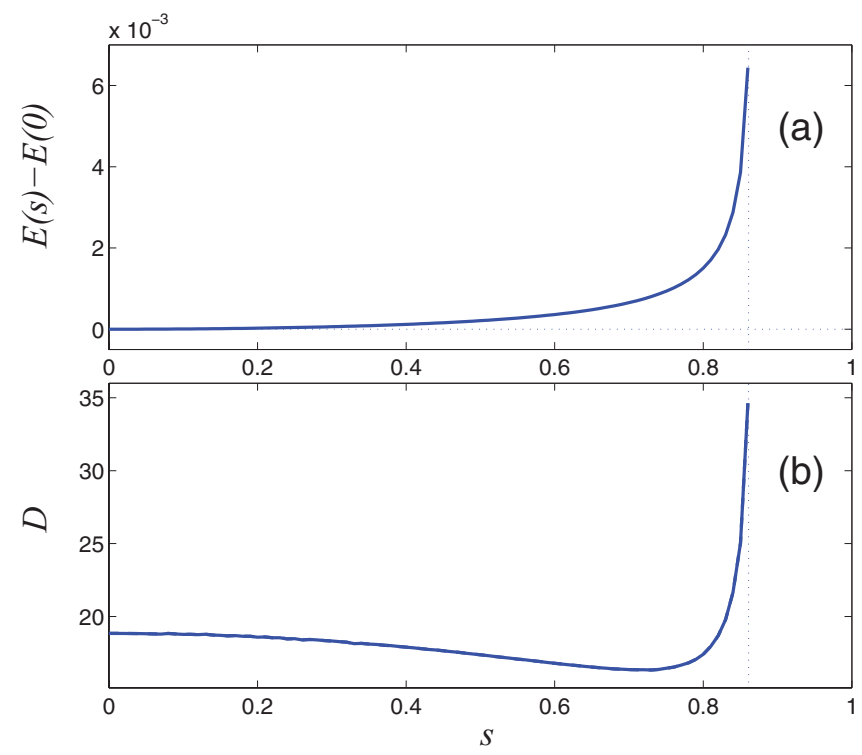

FIG. 9. (Color online) Dependence of energy $E$ and diameter $D$ on reduced velocity $\bar{s}$ at $\beta=1, r_{0}=1, \kappa=1$, and $a=1.7$.
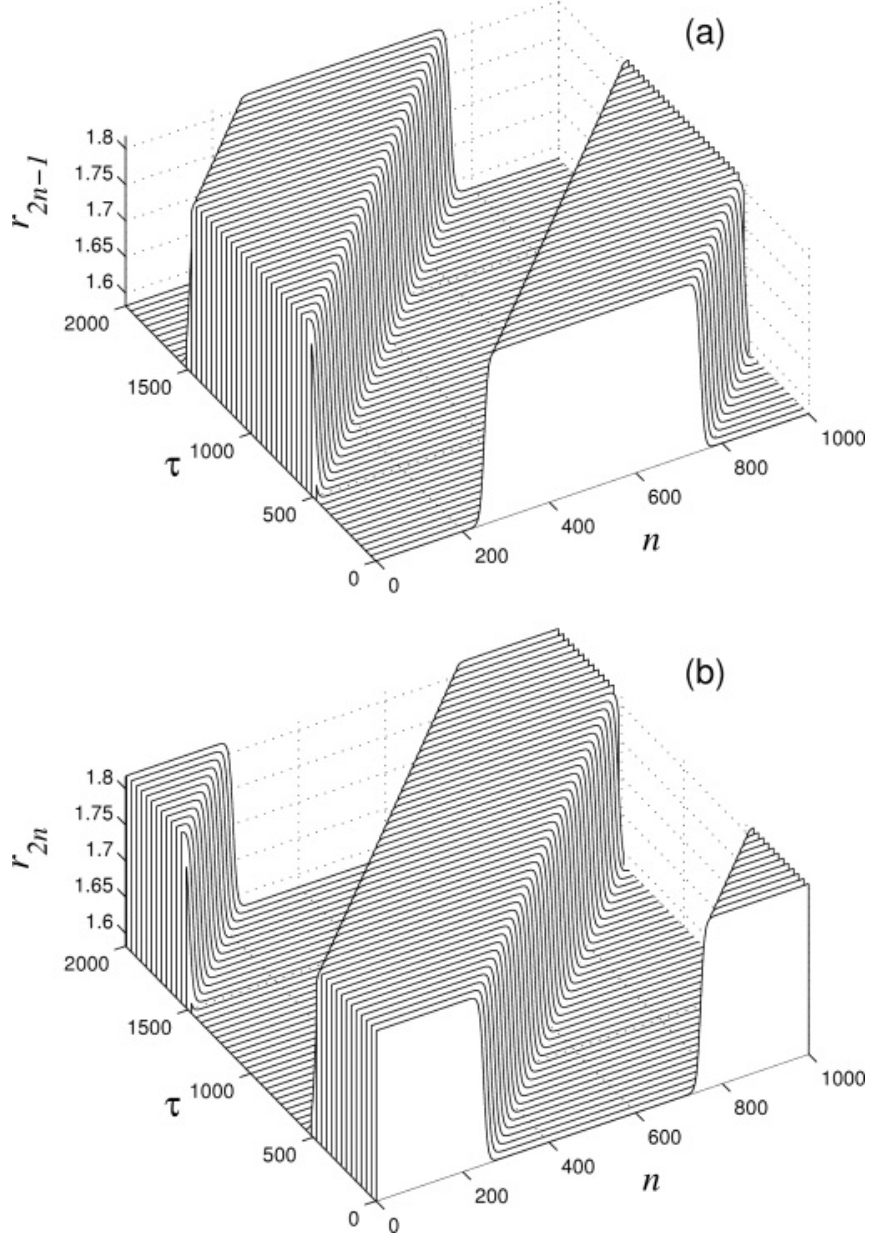

FIG. 10. Uniform motion of kink-antikink pair in stretched chain. Dependence of chain distribution of odd $r_{2 n-1}$ (a) and even $r_{2 n}$ (b) bond lengths on time $\tau$. Parameter values: $\beta=1, r_{0}=1, \kappa=1, a=$ 1.7 (lattice spacing of stretched chain), $\bar{s}=s / 2 a \sqrt{\kappa}=0.5$ (reduced soliton velocity).

Consider the dynamics of a kink-antikink pair in a cyclic chain consisting of $N$ sites. To this end, we integrate the system of the equations of motion (25) with the initial conditions

$$
\begin{aligned}
r_{n}(0) & =r_{n}^{0}, \quad \text { for } n=1,2, \ldots, 2 N ; \\
r_{2 n-1}^{\prime}(0) & =-s\left(r_{2 n+1}^{0}-r_{2 n-3}^{0}\right) / 4 a, \\
r_{2 n}^{\prime}(0) & =-s\left(r_{2 n+2}^{0}-r_{2 n-2}^{0}\right) / 4 a, \\
\text { for } \quad n & =1,2, \ldots, N,
\end{aligned}
$$

where $s$ is a soliton velocity and $\left\{r_{n}^{0}\right\}_{n=1}^{2 N}$ is a topological soliton solution with velocity $s$.

The numerical integration of the system (25) with the initial conditions (32) has shown that the topological solitons in the stretched chain are dynamically stable for all admissible velocities $s<s_{0}$. As illustrated by Fig. 10, the solitons move along the chain with a constant velocity without phonon radiation, completely retaining their initial shape.

Consider now the interaction of the solitons with opposite polarity under their collision. To this end, we integrate the 

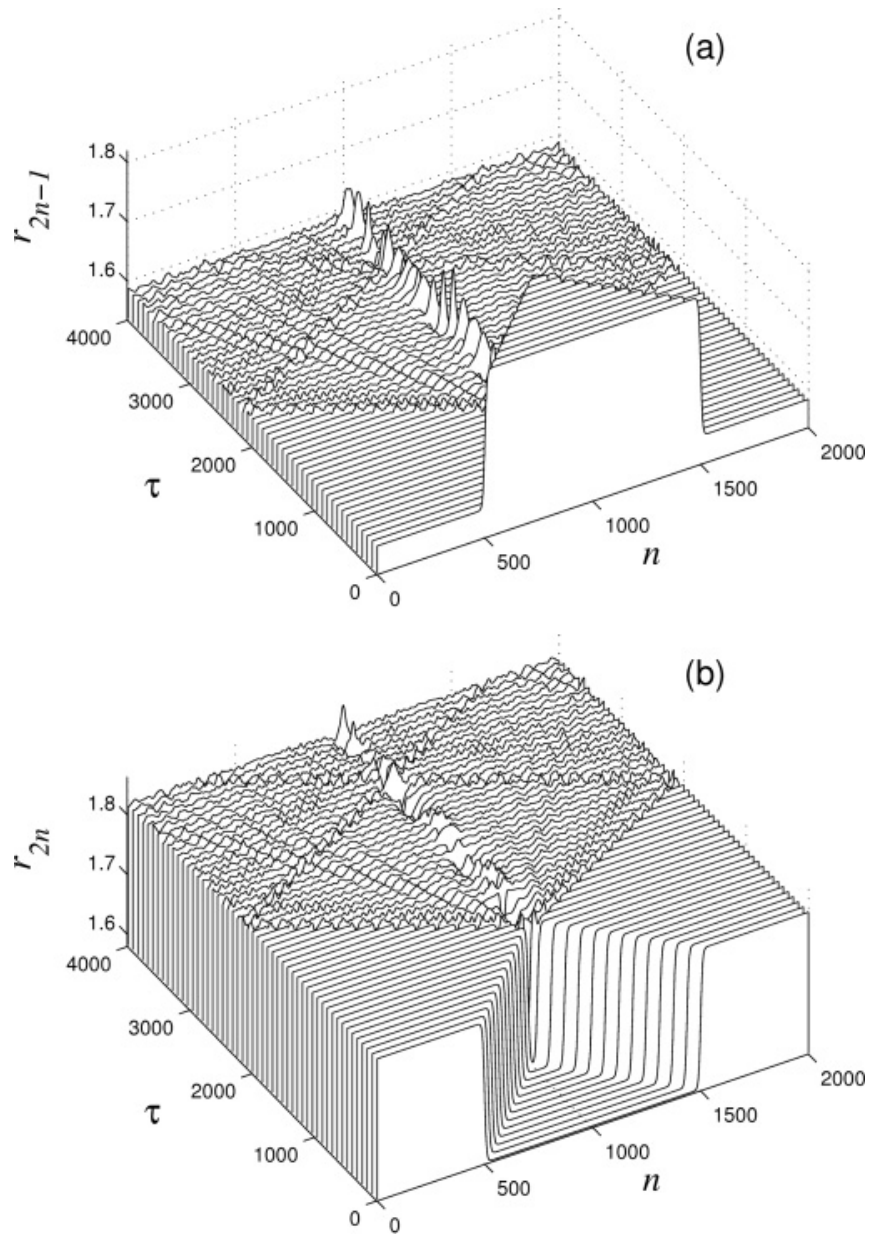

FIG. 11. Annihilation of two topological solitons with opposite polarity under collision in stretched chain. Dependence of the chain distribution odd $r_{2 n-1}$ (a) and even $r_{2 n}$ (b) bond lengths is shown. Parameter values: $\beta=1, r_{0}=1, \kappa=1, a=1.7$ (lattice spacing of stretched chain), $\bar{s}=s / 2 a \sqrt{\kappa}=0.5$ (reduced soliton velocity).

system (25) with the initial conditions

$$
\begin{aligned}
r_{n}(0) & =r_{n}^{0}, \quad \text { for } n=1,2, \ldots, 2 N ; \\
r_{2 n-1}^{\prime}(0) & =-s\left(r_{2 n+1}^{0}-r_{2 n-3}^{0}\right) / 4 a, \\
r_{2 n}^{\prime}(0) & =-s\left(r_{2 n+2}^{0}-r_{2 n-2}^{0}\right) / 4 a, \\
\text { for } \quad n & =1,2, \ldots, N / 2 ; \\
r_{2 n-1}^{\prime}(0) & =s\left(r_{2 n+1}^{0}-r_{2 n-3}^{0}\right) / 4 a, \\
r_{2 n}^{\prime}(0) & =s\left(r_{2 n+2}^{0}-r_{2 n-2}^{0}\right) / 4 a, \quad \text { for } \\
n & =N / 2+1, N / 2+2, \ldots, N .
\end{aligned}
$$

The numerical integration has shown that this interaction is inelastic. Thus, at the velocity $\bar{s}=0.5$, the collision results in the annihilation of solitons with opposite polarity. The collision is accompanied by intensive phonon radiation and leads to the appearance a breatherlike localized oscillation (see Fig. 11).

For $\kappa<\kappa_{0}$ the chain can be found in the ground state of three types, depending on the lattice spacing $a$. Under weak stretching $a \leqslant a_{1} \quad\left(1<a_{1}<a_{0}\right)$, the ground state is a uniformly stretched chain with equal bonds. At middle stretching $a_{1}<a<a_{2}\left(a_{2}>a_{0}\right)$, a part of the chain is found

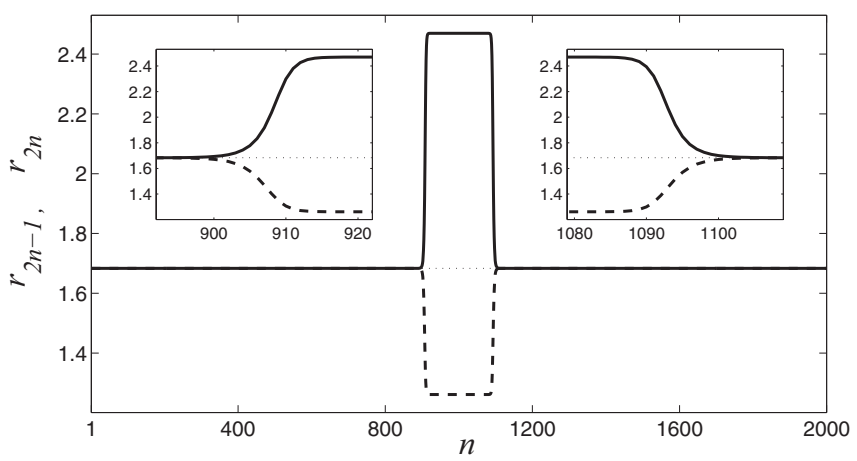

FIG. 12. Nonuniform stationary state of stretched cyclic chain with $a>a_{0}$ and $\kappa<\kappa_{0}\left(\beta=1, r_{0}=1, a=1.7, \kappa=0.2\right)$. Insets show that the edges of strongly stretched region of the chain have soliton shape (bond lengths $r_{2 n-1}, r_{2 n}$ smoothly depend on $n$ ).

in a weakly stretched homogeneous state with equal bonds and the spacing $a=a_{1}$, whereas the complementary part in a strongly stretched state with alternating bonds and the spacing $a=a_{2}$. For $a \geqslant a_{2}$ the whole chain is found in a homogeneous state with alternating bonds.

The numerical analysis confirms the existence of these three types of chain states. Thus, at $\beta=1, r_{0}=1, \kappa=0.2$, the critical values of the lattice spacing are $a_{0}=1+\ln 2=$ 1.6931, $a_{1}=1.6833, a_{2}=1.8655$. At $a=1.7$ the main part of the chain is found in the uniform state with equal bond lengths $a_{1}<a_{0}$, whereas the other part turns into the strongly stretched alternating state with period $a_{2}>a_{0}$ (see Fig. 12). This behavior of the chain under stretching can be explained by the nonconvexity of the function $E(a)$ (see Sec. III).

Figure 12 also illustrates that the edges of the strongly stretched region of the chain with the alternating structure have the form of smooth stairs describing a smooth transition of the chain from the state with equal bonds to the state with alternating weakly and strongly stretched bonds. The numerical simulations have shown that the strongly stretched region can propagate along the chain with a subsonic velocity completely retaining its shape (see Fig. 13). Therefore this transition region of the chain is a soliton describing the transition of only a part of the chain into the state with alternating bonds. As follows from this figure, the collision of these solitons does not result in their destruction, but it is accompanied by phonon radiation.

\section{EXAMPLES OF MOLECULAR CHAINS WHERE THE EXISTENCE OF STRETCHING SOLITONS IS POSSIBLE}

We have studied the simplified one-dimensional lattice model. Nevertheless, this study allows us to define a family of molecular systems with quasi-one-dimensional structure in which the effects related to the appearance of bistability under stretching are expected.

The stability condition of alternating states of the stretched chain (18) imposes an important constraint-the coupling constants of the first and the second neighbors have to be related through this inequality. This condition of commensurability of the energies of interaction cannot obviously be realized in the chains, where the nearest neighbors are coupled by strong 

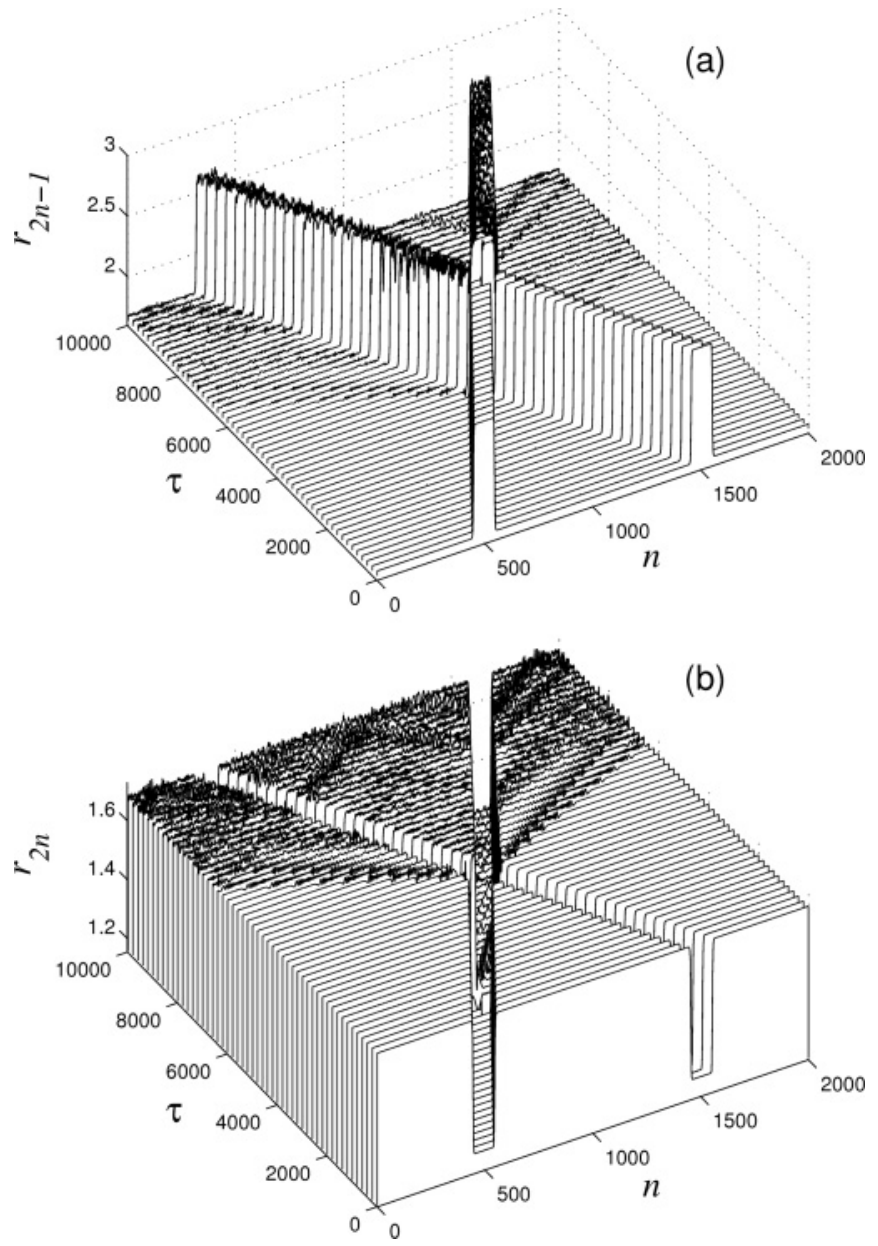

FIG. 13. Inelastic collision of two strongly stretched regions in cyclic chain with $a>a_{0}$ and $\kappa<\kappa_{0}\left(\beta=1, r_{0}=1, a=1.7, \kappa=\right.$ 0.2 , velocity is $\bar{s}=0.25)$. Dependence of chain distribution of odd $r_{2 n-1}$ (a) and even $r_{2 n}$ (b) bond lengths on time $\tau$ is present.

valent bonds, while the second neighbors by weak van der Waals interactions.

Note that the condition (18) provides the stability of a stretched chain only with respect to its longitudinal deformations. In the three-dimensional space, the instability of a stretched chain can be caused by other (orientational, bending, twisting, etc.) deformations. Therefore the commensurability condition in this case is not sufficient.

Consider a zigzaglike chain of hydrogen bonds as the first example. Hydrogen fluorides $\mathrm{HF}$, chlorides $\mathrm{HCl}, \mathrm{HBr}$, and $\mathrm{HI}$ $(\mathrm{H} X)$ at low temperatures have a crystalline structure formed by planar zigzaglike chains of hydrogen bonds [13-16]. Consider an isolated hydrogen-bonded chain $(\mathrm{H} X \cdots)_{\infty}$ consisting of two-atom molecules of fluoride $\mathrm{HF}$ and chloride $\mathrm{HCl}$.

The interaction of two-atom polar molecules $\mathrm{H} X$ is usually described by the 12-6-1 potential [17]

$$
U=\sum_{i_{1}=1}^{3} \sum_{i_{2}=1}^{3} \frac{q_{i_{1}} q_{i_{2}}}{r_{i_{1} i_{2}}}+4 \epsilon\left[\left(\frac{\sigma}{r}\right)^{12}-\left(\frac{\sigma}{r}\right)^{6}\right],
$$

with the seven free parameters: two Lennard-Jones parameters $\epsilon$ and $\sigma$, three charges $q_{1}, q_{2}$, and $q_{3}\left(q_{1}+q_{2}+q_{3}=0\right)$, lying on the line of valent bonds, and three distances $r_{1}, r_{2}$, and $r_{3}$, which assign the charge positions. Here $r_{i_{1} i_{2}}$ is the distance between the charge $q_{i_{1}}$ of the first molecule $\mathrm{H} X$ and the charge $q_{i_{2}}$ of the second molecule given in terms of $r_{1}, r_{2}$, and $r_{3}$.

The values of the parameters for potential (34) can easily be found using the data of the crystalline structure of $(\mathrm{HX})_{x}$ and ab initio calculations of the dimer $(\mathrm{H} X)_{2}$ [18]. We get

$$
\begin{aligned}
q_{1} & =-0.6397 e, \quad q_{2}=0.6159 e, \quad q_{3}=0.0238 e, \\
r_{1} & =0.25 \AA, \quad r_{2}=0.9075 \AA, \quad r_{3}=-1.575 \AA, \\
\epsilon & =0.00798 \mathrm{eV}, \quad \sigma=2.837 \AA
\end{aligned}
$$

for hydrogen fluoride HF and

$$
\begin{aligned}
q_{1} & =-0.3147 e, \quad q_{2}=-0.7974 e, \quad q_{3}=0.4827 e, \\
r_{1} & =1.296 \AA, \quad r_{2}=-0.275 \AA, \quad r_{3}=-0.838 \AA, \\
\epsilon & =0.0298 \mathrm{eV}, \quad \sigma=3.602 \AA
\end{aligned}
$$

for hydrogen chloride $\mathrm{HCl}$, where $e$ is the electron charge.

In the plane of the zigzaglike chain $(\cdots \mathrm{H} X \cdots)_{\infty}$, the position of each molecule $\mathrm{HX}$ is given by the coordinates $x$ and $y$ of the center of the heavy molecule $X$ and the angle $\phi$, which shows the direction (orientation) of the molecule $\mathrm{H} X$. The detailed description of the quantum-mechanical model of this zigzaglike structure is given in [19]. For hydrogen fluoride, the zigzag angle is $\alpha=119.5^{\circ}$, the longitudinal lattice spacing is $l_{x}=2.167 \AA$, the distance between the nearest molecules is $\rho_{0}=2.509 \AA$, the direction of each chain molecule differs from the zigzag line only by the angle $\varphi_{0}=01.21^{\circ}$. (The parameters for hydrogen chloride are $\alpha=93.6^{\circ}, l_{x}=2.692 \AA$, $\rho_{0}=3.694 \AA, \varphi_{0}=0.95^{\circ}$.) In equilibrium, the energy of interaction of the nearest molecules is $E_{1}=0.2339 \mathrm{eV}$ and the energy of interaction of the second-neighboring molecules is $E_{2}=0.0312 \mathrm{eV}\left(E_{1}=0.0890 \mathrm{eV}, E_{2}=0.0165 \mathrm{eV}\right)$.

Here the commensurability condition (18) of the energy of interaction of the first and second neighbors is fulfilled. Thus for the chain of molecules of hydrogen fluoride $E_{2} / E_{1}=$ $0.133>1 / 8$ and for hydrogen chloride $E_{2} / E_{1}=0.185>$ $1 / 8$. However, the analysis of spectrum behavior under the chain stretching has shown that the stability of a uniformly stretched state of the chain disappears before reaching the point of inflection of the effective potential of longitudinal stretching. In the case of HF, for the point of inflection the longitudinal lattice spacing of the zigzag is $a_{0}=2.67 \AA$, while the stability of the chain disappears already at the longitudinal lattice spacing $a_{1}=2.625 \AA$ (for $\mathrm{HCl} a_{0}=3.60 \AA, a_{1}=$ $3.48 \AA$ ). For the longitudinal spacing of the zigzag $a \geqslant a_{1}$, the chain becomes unstable with respect to the bending long-wave phonons, i.e., the bending instability of the chain takes place. Note that as regards the rest of the (longitudinal and orientational) phonons, the chain remains stable under the stretching $a>a_{0}$ ). Thus the bending instability of zigzaglike chains does not admit the existence of the topological solitons of stretching. The maximum possible stretching of the chain can be defined as the ratio $a_{1} / l_{x}$. For the chain $(\cdots \mathrm{HF} \cdots)_{\infty}$ the maximum stretching is $21 \%$, while for the chain $(\cdots \mathrm{HCl} \cdots)_{\infty}$ it is $29 \%$.

The similar bending instability of the chain is observed under stretching the transzigzag of the polyethylene macromolecule $\left(-\mathrm{CH}_{2}-\right)_{n}$. The analysis of the linear dynamics of the planar zigzag of the chain within the model studied 
in Refs. [19,20] has shown that the strained chain is stable under stretching (the longitudinal lattice spacing of the zigzag) $a \leqslant a_{0}$, where the critical value $a_{0}=1.745 \AA$ corresponds to the point of inflection of the effective potential of longitudinal stretching. However, under stretching $a>a_{0}$ the chain becomes unstable with respect to the bending oscillations of the chain. Here the maximum possible stretching of the chain is $37 \%$ (in equilibrium the longitudinal lattice spacing of the zigzag is $l_{x}=1.276 \AA$ ). Here the bending instability is caused by the fact that the interaction of the second neighbors in the transzigzag occurs only because of the deformation of the valent angles CCC and it does not depend directly on the distance between them. The bending of the chain does not allow to break it without deforming the valent angles.

Thus, in a zigzaglike polyethylene macromolecule, the energy of interaction of the second neighbors is of the same order as the energy of interaction of the first neighbors, but the formation of bistable ground states is impossible due to the bending instability of the strongly stretched chain. For the absence of this instability the angles of a polymer chain have to possess a sufficiently strong nonvalent interaction of the second neighbors. The molecular groups of polyethylene $\mathrm{CH}_{2}$ do not possess the interaction of this type. However, the radicals $\mathrm{CHR}$ with sufficiently long chains can provide this interaction. The polyolefine macromolecules $\left(-\mathrm{CH}_{2}-\right.$ $\mathrm{CHR}-)_{n}$ : polypropylene $\left(R=\mathrm{C}_{3} \mathrm{H}_{7}\right)$, polystyrene containing benzol rings in radicals $\left(R=\mathrm{C}_{6} \mathrm{H}_{5}\right)$ and polyvinylcarbazole have the required stability structure. In these macromolecules with strongly interacting side radical groups $R$ under the strong stretching of the chain, the topological solitons can exist.

The existence of the topological solitons of stretching can be expected also in the DNA double helix. Here the conformational interaction of neighboring sites of the sugarphosphate lattice plays the role of the first-neighbor coupling, whereas the stacking interaction of the neighboring purine and pyrimidine bases can be considered as the second-neighbor interaction. The experiments on stretching a single DNA molecule [1-4] exhibit the presence of a specific constant region (plateau) in the force-stretching diagram [5]. At strength about $65 \mathrm{pN}$ the nontypical behavior is exhibited: the molecule becomes elongated at constant force up to 1.7 of its contour length. At further stretching the force again begins to grow. A similar behavior under stretching exhibits also $\alpha$ helices of protein [10].

Within our model this behavior will take place under a weak second-neighbor interaction, when $K<k_{0}$. Here, in a certain interval of lengthening, the stretching occurs according to the two-phase scenario, when one part of the chain is found in a weakly and the other one in a strongly stretched state. In the force-stretching diagram a constant region (plateau) appears due to the stretching of the chain because of increasing only the portion of its strongly stretched part. Obviously, here the topological solitons describing the transition from the weakly to the strongly stretched phases of the chain have to exist.

\section{CONCLUSIONS}

The study carried out in this paper shows under the stretching of molecular chains the conformational changes of these chains can occur that result in different ground states. The transition regions between these states can be described as topological solitons. In the simplest model with the nearest-neighbor interaction of the Morse-like type and the second-neighbor harmonic interaction, it is shown that under the chain stretching the ground state is realized as a regular configuration with alternating bonds ("long-short"). In this case, the chain can be found in two degenerate ground states admitting the existence of topological solitons that describe the chain transition from the state "short-long bond" into the state "long-short bond." This situation is possible for the molecular chains with sufficiently strong interaction of the second neighbors. With weak interaction, the chain stretching leads to the appearance of one region with weakly and the other one with strongly stretched bonds. As a result of this nonuniform stretching, the presence of a broad plateau in the force-stretching diagram of DNA double helix and protein $\alpha$ helix can be explained. The boundary between the weakly and strongly stretched phases of the chain can also be described as a topological soliton.

Finally, it should be noticed that the models with the secondneighbor coupling being responsible for the stabilization of homogeneous bistable ground states have been studied earlier [19,21-24]. However, in these (diatomic) models, the effect of switching or controlling bistability by external forcing has not been considered. The bistability here has been attained intrinsically due to the repulsive interaction in the heavy-ion sublattice.

\section{ACKNOWLEDGMENTS}

A.V.Z. acknowledges the partial financial support from the Ukrainian State Grant for Fundamental Research. Both of us (A.V.S. and A.V.Z.) would also like to express gratitude to the MIDIT Center and Department of Informatics and Department of Physics of the Technical University of Denmark for partial financial support and hospitality.
[1] P. Cluzel, A. Lebrun, A. Heller, R. Lavery, J.-L. Viovy, D. Chatenay, and F. Caron, Science 271, 792 (1996).

[2] S. B. Smith, Y. Cui, and C. Bustamante, Science 271, 795 (1996).

[3] M. C. Williams, K. Pant, I. Rouzina, and R. L. Karpel, Spectroscopy 18, 203 (2004).
[4] M. J. McCauley and M. C. Williams, Biopolymers 91, 265 (2008).

[5] C. Bustamante, S. B. Smith, J. Liphardt, and D. Smith, Curr. Opin. Struct. Biol. 10, 279 (2000).

[6] I. Schwaiger, C. Sattler, D. R. Hostetter, and M. Riff, Nat. Lett. 1, 232 (2002). 
[7] R. Afrin, I. Takahashi, K. Shiga, and A. Ikai, Biophys. J. 96 1105 (2009).

[8] F. Ritort, J. Phys.: Condens. Matter 18, R531 (2006).

[9] L. I. Manevitch, L. S. Zarkhin, and N. S. Enikolopian, J. Appl. Polym. Sci. 39, 2245 (1990).

[10] F. C. Zegarra, G. N. Peralta, A. M. Coronado, and Y. Q. Gao, Phys. Chem. Chem. Phys. 11, 4019 (2009).

[11] P. L. Christiansen, A. V. Savin, and A. V. Zolotaryuk, Phys. Rev. B 54, 12892 (1996).

[12] P. L. Christiansen, A. V. Savin, and A. V. Zolotaryuk, J. Comput. Phys. 134, 108 (1997).

[13] A. Anderson, B. H. Torrie, and W. S. Tse, J. Raman Spectrosc. 10, 148 (1981)

[14] M. Atoji and W. N. Lipscomb, Acta Crystallogr. 7, 173 (1954).
[15] M. Ghelfenstein and H. Szwarc, Mol. Cryst. Liq. Cryst. 14, 273 (1971).

[16] D. F. Smith and J. Overend, J. Chem. Phys. 54, 3632 (1971).

[17] M. E. Cournoyer and W. L. Jorgensen, Mol. Phys. 51, 119 (1984).

[18] A. V. Nemukhin, Zhurn. Fiz. Khimii 66, 4 (1992) (in Russian).

[19] A. V. Savin, L. I. Manevich, P. L. Christiansen, and A. V. Zolotaryuk, Phys. Usp. 42, 245 (1999).

[20] L. I. Manevitch and A. V. Savin, Phys. Rev. E 55, 4713 (1997).

[21] A. V. Zolotaryuk, St. Pnevmatikos, and A. V. Savin, Physica D 51, 407 (1991).

[22] Y. S. Kivshar, Phys. Rev. A 43, 3117 (1991).

[23] A. V. Zolotaryuk, M. Peyrard, and K. H. Spatschek, Phys. Rev. E 62, 5706 (2000).

[24] V. M. Karpan, Y. Zolotaryuk, P. L. Christiansen, and A. V. Zolotaryuk, Phys. Rev. E 70, 056602 (2004). 